\title{
Technical solutions for simultaneous MEG and SEEG recordings: towards routine clinical use
}

\author{
Badier JM ${ }^{1}$, Dubarry AS ${ }^{1}$, Gavaret $M^{1,2}$, Chen $S^{1}$, Trébuchon $\mathrm{AS}^{1,2}$, Marquis $\mathrm{P}^{1}$, Régis \\ $\mathbf{J}^{1,3}$, Bartolomei $\mathrm{F}^{1,2}$, Bénar $\mathrm{CG}^{1 *}$, Carron $\mathrm{R}^{1,3}$. \\ ${ }^{1}$ Aix Marseille Univ, Inserm, INS, Institut de Neurosciences des Systèmes, Marseille, France \\ ${ }^{2}$ APHM, Timone hospital, Clinical Neurophysiology, Marseille, France \\ ${ }^{3}$ APHM, Timone hospital, Functional and Stereotactic Neurosurgery, Marseille, France \\ *: equally contributing authors
}

E-mail: christian.benar@univ-amu.fr

\begin{abstract}
The simultaneous recording of intracerebral EEG (stereotaxic EEG, SEEG) and magnetoencephalography (MEG) is a promising strategy that provides both local and global views on brain pathological activity. Yet, acquiring simultaneous signals poses difficult technical issues that hamper their use in clinical routine. Our objective was thus to develop a set of solutions for recording a high number of SEEG channels while preserving signal quality. We recorded data in a patient with drug resistant epilepsy during presurgical evaluation. We used dedicated insertion screws and optically insulated amplifiers. We recorded 137 SEEG contacts on 10 depth electrodes (5 to 15 contacts each) and 248 MEG channels (magnetometers). Signal quality was assessed by comparing the distribution of RMS values in different frequency bands to a reference set of MEG acquisitions. It was excellent for both MEG and SEEG; for MEG, it was comparable to that of MEG signals without concurrent SEEG. Discharges involving several structures on SEEG were visible on MEG, whereas discharges limited in space were not seen at the surface. SEEG can now be recorded simultaneously with whole-head MEG in routine. This opens new venues, both methodologically for understanding signals and improving signal processing methods, and clinically for future combined analyses.
\end{abstract}

Keywords: MEG; SEEG; epilepsy; simultaneous recordings 


\section{Introduction}

Magnetoencephalography (MEG) allows recording non-invasively and with millisecond accuracy the complex spatio-temporal dynamics of brain networks. It has proven particularly efficient in presurgical mapping for epilepsy (Stefan et al., 2011), due in part to the low influence of skull conductivity on the resulting signals (Lopes da Silva and Van Rotterdam, 2005). However, MEG remains a surface measure, thus bearing intrinsic limitations. Firstly, MEG - as scalp EEG - needs to solve an inverse problem for estimating actual cortical activity based on surface signals. Many algorithms have been proposed to solve this ill-posed problem (Baillet et al., 2001), with different assumption leading to different solutions. There is therefore a pressing need for a "ground truth" that would enable comparing different methods based on independent recordings. Secondly, neuronal activity needs to be summed over large areas of cortex in order to produce visually detectable activity at the surface. As a consequence, the best strategies for inferring neuronal activity, and in particular that of deep sources (such as amygdala, hippocampus or thalamus), remain to be defined (Wennberg et al., 2011).

In contrast, invasive recordings such as stereo-electroencephalography (SEEG) performed in the setting of presurgical evaluation of epilepsy provide a unique opportunity for recording directly within brain structures - either superficial or deep - with high spatial specificity and high signal to-noise ratio. Still, only a limited set of electrodes can be placed within the brain (typically between 10 and 15), providing only a limited spatial sampling of the brain volume.

An optimal strategy is thus to record simultaneously signals from MEG and from invasive electrodes. Such recordings permit to benefit from the advantages of the two modalities, providing a view on brain pathological discharges that is both local (SEEG) and global (MEG) (Gavaret et al., 2016). So far, only a few studies have presented simultaneous MEG and SEEG recordings (Santiuste et al., 2008; Kakisaka et al., 2012; Dubarry et al., 2014) or simultaneous MEG and subdural electrocorticography (Mikuni et al., 1997; Sutherling et al., 2001). These seminal studies emphasized the technical difficulties that are inherent to simultaneous acquisitions. The first difficulty is of a mechanical nature. The post-operative bandage wrapped around the head and the SEEG insertion screws can result in increased volume, possibly preventing the head from fitting in the MEG helmet. The second difficulty arises from possible artefacts of EEG recording system onto the MEG signals (Dubarry et al., 2014; Rampp et al., 2010). SEEG fixation and connector systems are a possible source of noise on the MEG, as well as the possible current leaks from amplifiers onto SEEG leads. Because of these constraints, or simply because of limitations of number of recording points or EEG channel number, previous simultaneous studies only recorded a limited number of channels. Current clinical practice is based on multiple lead recordings with a high number of channels (up to 200), which requires solving technical issues first in order to have high spatial sampling for both MEG and SEEG. 
In the present case study, we introduce technical solutions - both at the surgical procedure level and at the signal acquisition level - that allowed recording signals of the same quality as that obtained in separate sessions. This resulted in high spatial sampling (137 SEEG contacts and 248 MEG channels) with very limited mechanical constraints.

\section{Methods}

\subsection{Patients information}

Both patients suffered from intractable epilepsy and underwent an SEEG exploration for presurgical evaluation. Patient 1 is a 19 -years-old woman with drug resistant cryptogenic left posterior cortex epilepsy. Seizures were characterized initially by an elementary visual hallucination (white or black ball, moving from left to right), then a head deviation to the right, a jargon and a secondary generalized tonic-clonic period in some seizures. Brain MRI was normal. Patient 2 is a 43 years old woman, right-handed, with drug resistant disabling left temporo-parietal epilepsy (cavernous angioma located in the left superior temporal gyrus that was previously resected in another hospital). She presented ictal aphasia, right arm jerks with some post ictal right arm motor deficit. An SEEG implantation exploring the left temporal and parietal regions was performed, with a total of 10 depth electrodes (Alcis, Besançon, France, ref. 2069-EPC) constituted of 5 to 15 contacts each. The patients gave written consent for simultaneous recordings, this research has been approved by the relevant Institutional Review Board (Comité de Protection des Personnes, Sud-Méditerranée I, ID-RCB 2012A00644-39).

\subsection{Surgical apparatus}

Patient 1 was implanted with standard insertion screws (Alcis, Besançon, France, ref. 2023VG) Patient 2 was implanted using dedicated small insertion screws (Alcis, Besançon, France, ref. 2023-VG-C-10;15;20;25;30) that enabled to minimize the bulk of screws and leads around the head (Figure 1). This is of utmost importance for the global head volume to be kept compatible with the MEG helmet. The use of these dedicated screws only slightly alters the surgical technique. Dedicated screwdrivers are required and the calculation of the needed electrode length (i.e., the distance between the target point and the plastic stop at the level of the external part of the insertion screw) is slightly different. It must indeed take into account and compensate for the 3 to $4 \mathrm{~mm}$ of the electrode length that bends at right angle at the outer level of the insertion screw. A-P (anteroposterior) X-Rays (Teleradiographs ) are systematically used to check the distance between the midline and the target point as for the classical technique. If required, the electrode can be inserted one or two millimeters deeper to reach the desired target. Specific tools are also needed to take out the screws when the exploration has enabled to gather sufficient data. The leads are flexible enough to be bent at right angle at the outer level of the insertion screws. Thus, the bulk of the insertion screws and leads 
together remains limited to less than $5 \mathrm{~mm}$ above skin level (Figure 1). Finally, head is wrapped in a light bandage.

\subsection{MEG-SEEG recordings}

For both patients MEG data were recorded with a whole head 4D system (248 magnetometers). Patient was set in supine position. For the SEEG, patient 1 was recorded with a 4D EEG device. 72 contacts were recorded from 13 electrodes. Patient 2 was recorded with optically insulated and battery operated amplifiers (256 channels BrainAmp, Brain Products GmbH, Munich, Germany) installed in the shielded room. A total of 134 depth electrode contacts were recorded, plus 2 EOG channels and 2 ECG channels. For both patients recordings were monopolar, with a mastoid electrode reference for patient 1 and an external contact reference from one SEEG electrode located in either the skull or the scalp for patient 2. Ground was obtained from an external SEEG contact. The head bandage was rearranged so that cables were going out in the occipital areas. The resulting cable length from the head to the connectors was about $25 \mathrm{~cm}$. For patient 1 the extension connectors were placed on the patient's shoulder. For patient two, the SEEG connectors were placed as far as possible from the patient head, lightly taped against the helmet in order to ensure that they would not move (figure 2). The head box was placed approximately $80 \mathrm{~cm}$ from the patient head and the amplifier/acquisition system was located on the ground near the patient's feet (Figure 2). Because a separate system have been used to record the SEEG signal for patient 2, regular triggers with small time jitters (range 3000$3500 \mathrm{~ms}$ ) were sent to both SEEG and MEG systems. Signals were co-registered offline based on these triggers with in-house code written in Matlab (Mathworks, Naticks, MA).

\subsection{Quantification of signal quality}

The quality of the simultaneous recording was compared to a series of 15 resting-state MEG-only datasets obtained in patients during routine presurgical evaluation of epilepsy, as well as the dataset presented in (Dubarry et al., 2014). For each dataset and each MEG channel, we computed the spectrum. We also computed the root mean square values (RMS) in two bands, $0.5-2 \mathrm{~Hz}$ and $13-60 \mathrm{~Hz}$, based on the spectral analysis and on a previous study (Dubarry et al., 2014). The RMS values of each channel (either in the new setup, "new", or in the previous setup, "old") were z-scored with respect to the RMS values across the reference datasets in the same channel:

$$
z_{\text {chan }}=\frac{r m s_{\text {chan }}-\frac{1}{n} \sum_{i=1}^{n} r m s_{c h a n}^{i}}{\sqrt{\frac{1}{n} \sum_{i=1}^{n}\left(r m s_{\text {chan }}^{i}\right)^{2}}}
$$

with $n$ number of reference datasets, and chan channel number. 


\section{Results}

Figure 1 shows the 3D reconstructions obtained from CT scans. In a) one can see the extra cephalic space that is occupied by the screws. Such a bilateral exploration would most of the time make impossible the simultaneous recording although it was possible this time thanks to the small head of the patient. b) and c) show the reconstructions for the side and rear view respectively with the new low profile elements. The resulting oversize is only due to the bandage of protection that is comparable to the space that would occupy an EEG cap.

We present in Figure 3 an example of simultaneous recording presenting interictal discharges for patient 2, after co-registration of MEG and SEEG traces in the same time frame. Only a subset of channels are shown (24 out of 248 for MEG, 23 out of 134 for the SEEG), selected to be representative of the interictal activity on the two modalities. Discharges involving several structures as visible on SEEG bipolar montage were visible on the MEG signals (marked by green arrows), whereas discharges limited in space (marked by red arrows) were not visually detectable on MEG signals. The topography at the peak of the MEG interictal spike clearly shows a dipolar pattern, suggesting a source in the left hemisphere, which is the side of SEEG implantation.

We obtained signals of excellent quality for both MEG and SEEG, indicating that SEEG recording did not interfere with MEG acquisition. Figure 4 show topographies of the power spectral density frequency maps for the two patients. In patient 1 (data from (Dubarry et al., 2014)), noisier topographies are seen, both in the low frequency range $(0.2-1 \mathrm{~Hz})$ and in the high frequency range (30$50 \mathrm{~Hz}$ ). In the previous study, low frequency noise was interpreted to arise from respiratory artefacts to the fact the connector was on the patient shoulder, thus moving with breathing (Figure 1b in (Dubarry et al., 2014)). The high frequency noise was observed at the level of the SEEG fixations (Figure 1d in (Dubarry et al., 2014)). It is likely that this artefact was due to current leakage from the previously used amplifier, which is suppressed in the new setup.

Figure 5 presents the distribution of z-scores of RMS energies across channels, for two datasets (from this study, labelled as "new', and from the (Dubarry et al., 2014) study, labelled as "old"). In the low frequency band $(0.5-2 \mathrm{~Hz})$, the "old" dataset shows many values extending outside the $\mathrm{z}= \pm 2$ lines, including high intensity outliers (red crosses). In the high frequency band (13-100 Hz), the old setup shows high intensity outliers corresponding to the channels with high intensity artefacts.

\section{Discussion}

The first simultaneous electrophysiology/MEG recordings were performed with electrocorticography (ECoG), in a limited number of subjects (Mikuni et al., 1997; Sutherling et al., 2001). Moreover, ECoG is a method that is designed for recording at the brain surface, without direct sampling of deep structures. A few studies have presented simultaneous intracerebral/MEG recordings (Santiuste et al., 2008; Kakisaka et al., 2012; Dubarry et al., 2014; Dalal et al., 2009; Wennberg et al., 2011; Gavaret et 
al., 2016), mentioning the difficulties inherent to such recordings. The first difficulty is to get the subject head inside the sensor - a constraint that is not applicable to EEG (Koessler et al., 2015). We show that dedicated SEEG insertion screws significantly cut down the added volume, thereby allowing the vast majority of patients to fit in the MEG helmet.

The second difficulty is to obtain signals of sufficient quality. A previous study from our group has shown influence of the SEEG amplifiers on MEG signal quality, in both high and low frequencies (Dubarry et al., 2014). Low frequency artefacts can arise from connector movements induced by breathing, whereas high frequency noise can be produced by the SEEG recording system together with cables and adaptors (Rampp et al., 2010). Most previous studies used band pass filtering to improve signal quality that limits further investigation of the use of high frequency analysis in ripples/fast ripples bands (Urrestarazu et al., 2007). Here, we show unfiltered signals of very high quality, both in SEEG (that benefits from the shielded room) and in MEG.

The third difficulty - related to signal quality - is the adequate spatial sampling. The first SEEG-MEG study reported only on one SEEG electrode, the electrode where seizure onset was obtained (Santiuste et al., 2008). This is not sufficient for describing network properties of epileptic discharges across different brain regions (Malinowska et al., 2014). Preserving the spatial sampling provided by a given SEEG implantation increases the clinical interest of simultaneous recordings, which can provide both local and global views of pathological activities (Kakisaka et al., 2012). In terms of MEG sensitivity, the SEEG sampling can allow to determine whether a discharge only arises in deep structures or jointly with other neocortical regions (Koessler et al., 2015). Here, we present a setup that permits recording up to 256 channels without compromising signal quality.

\section{Conclusion}

We present in this report technical solutions for recording SEEG simultaneously with whole-head MEG in routine. With this setup, the constraints concerning head size are very limited, comparable to those in simultaneous recordings of MEG and surface EEG. Moreover, all available SEEG channels can be recorded and there is no additional limitation to the classical MEG requirements regarding magnetic compatibility.

Such recordings open a unique opportunity to better understand the links between surface and depth signals, and to optimize signal processing techniques. Furthermore, they make possible a combined analysis at two levels of recordings, local (SEEG) and global (MEG). In particular, they will enable characterizing networks between deep structures and neocortex, for both interictal spikes and oscillations. Applications of these new strategies should arise in both fundamental neurosciences and clinics. 


\section{Figures}

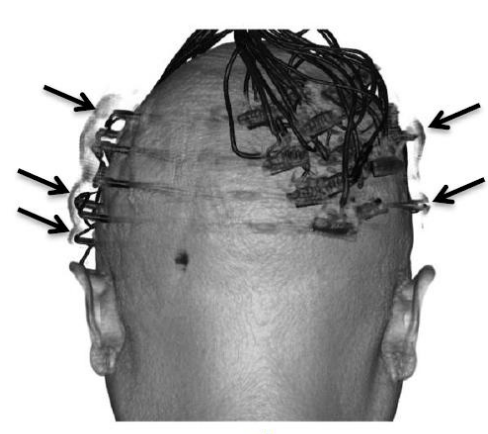

a

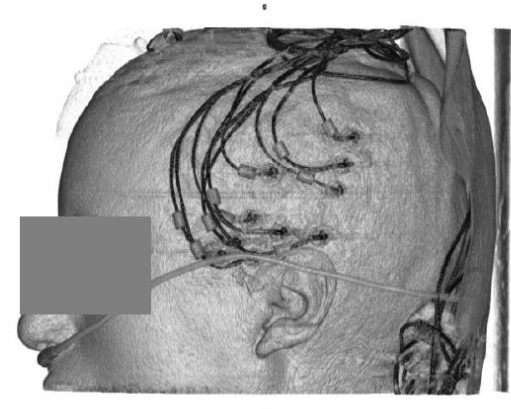

b

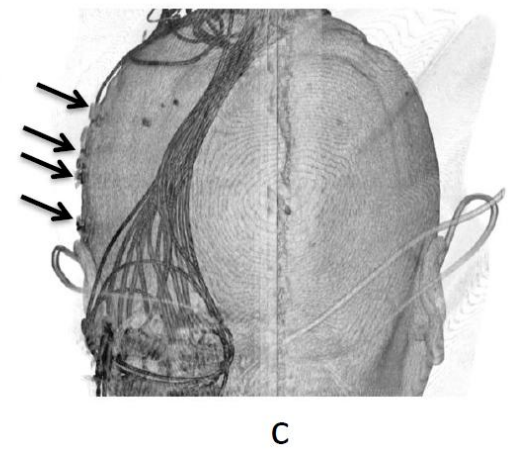

C

Figure 1: Comparison of the depth electrode holding screws for the two patients. Reconstructions were obtained from post implantation CT scans (OsiriX software). a) Rear view of the reconstruction for the patient 1 (with long screws). b) and c) reconstruction for short screws on profile and rear view respectively for patient 2 . 

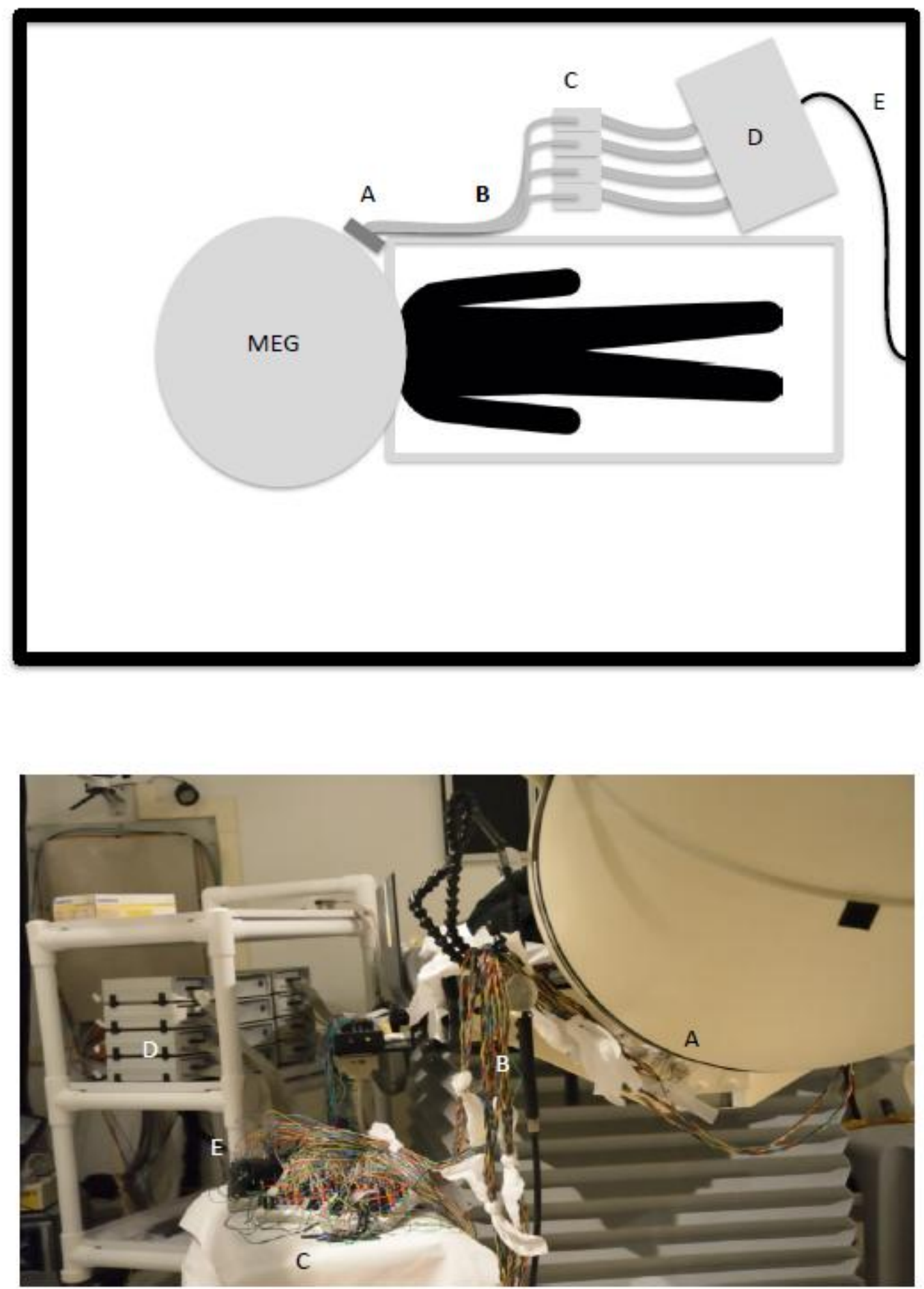

Figure 2: Experimental setup within the shielded room of the MEG. a) Diagram and b) photography. A. Depth electrode connectors. B. Cable extensions. C. Head boxes. D. Battery operated amplifiers. E. Optical fiber for the transmission of the data outside the shielded room. 
a)

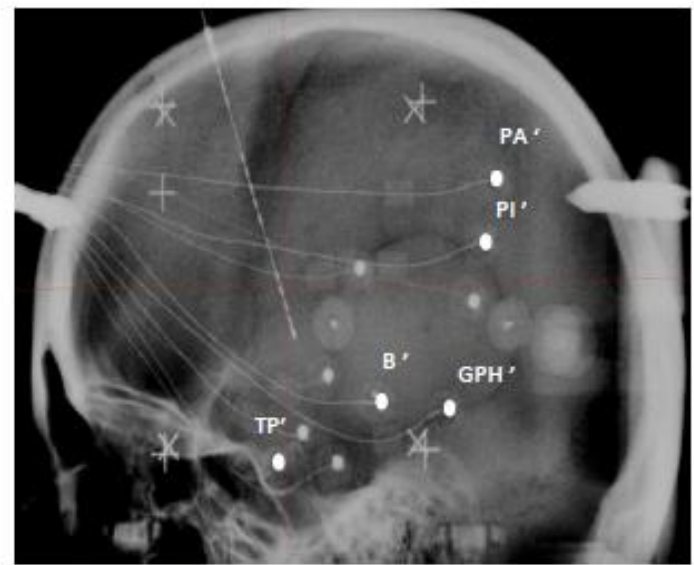

b)

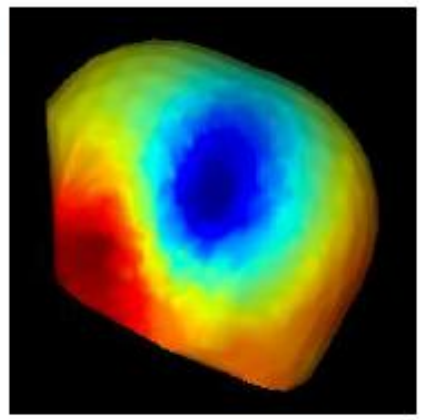

c)
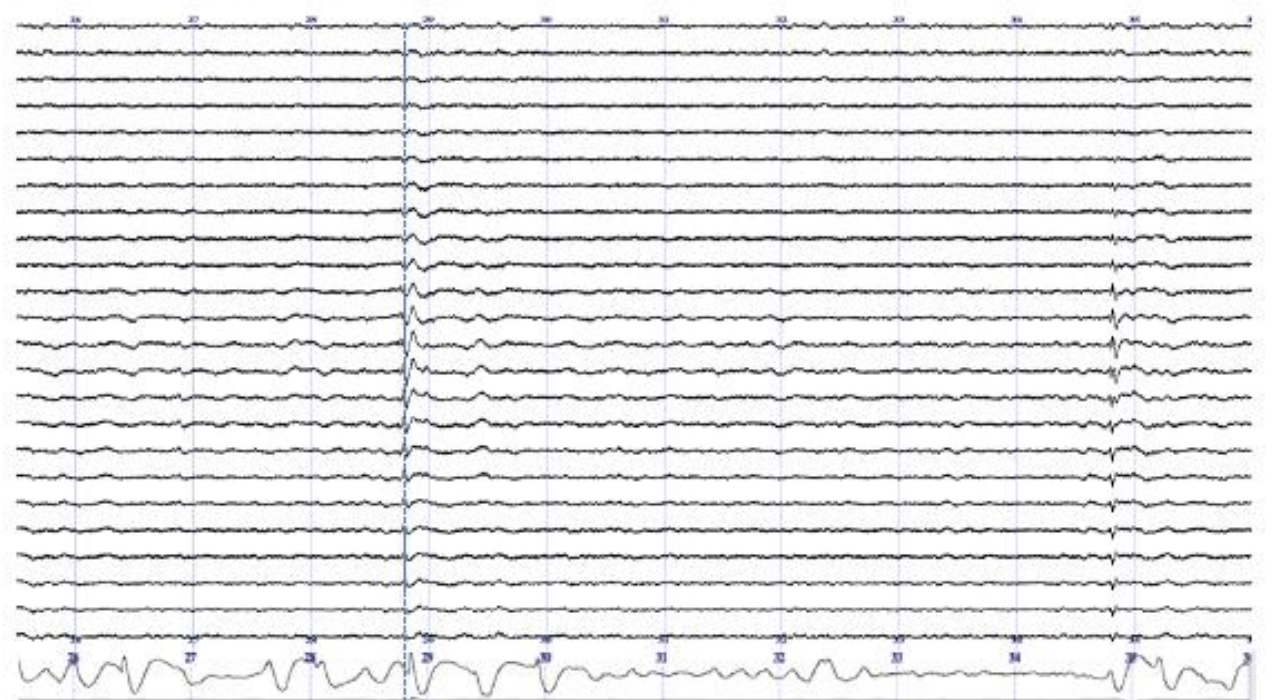

$\cdot 1$

$\mathrm{GPH}^{\prime}$
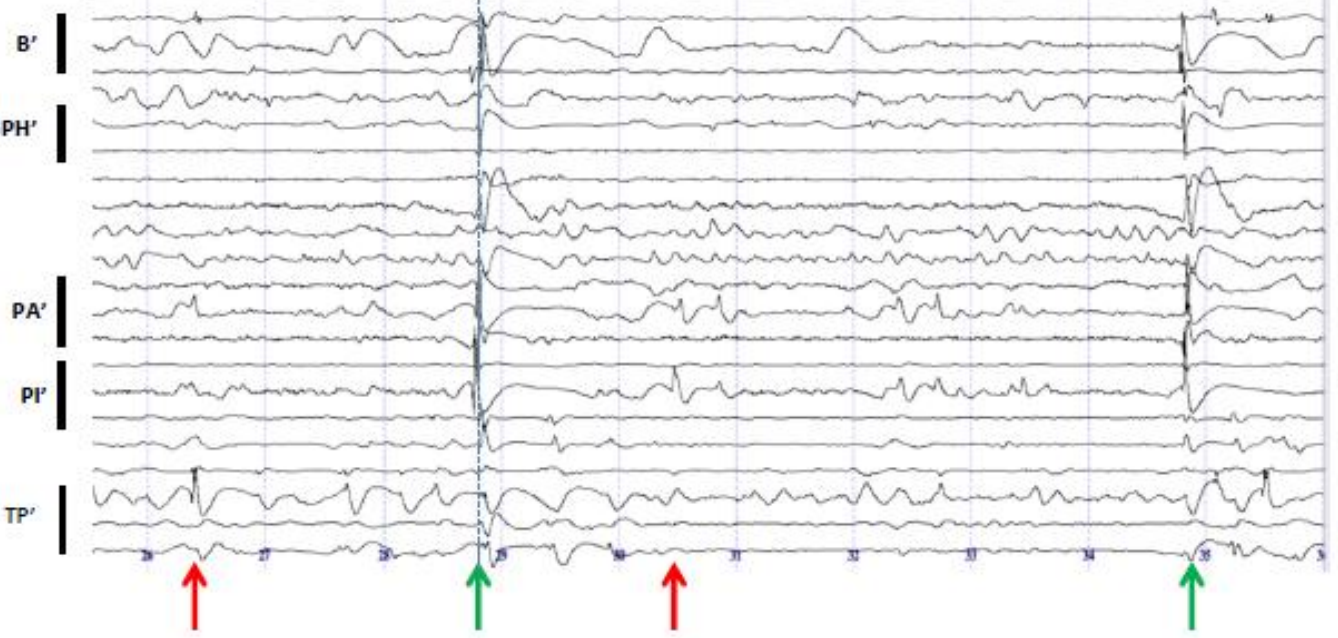

Figure 3: Example of simultaneous recording. a) SEEG implantation. Only the names of the electrodes presented below are shown. b) MEG amplitude mapping corresponding to the first visible interictal spike. c) Simultaneous MEG (top) and SEEG (bottom) data. Interictal spikes involving several structures (B', GPH', PA', PI' leads) are visually detectable in MEG signals (green arrows), whereas interictal spikes limited spatially (limited to PA' and PI' medial leads) are not visually detectable in MEG signals (red arrows). 

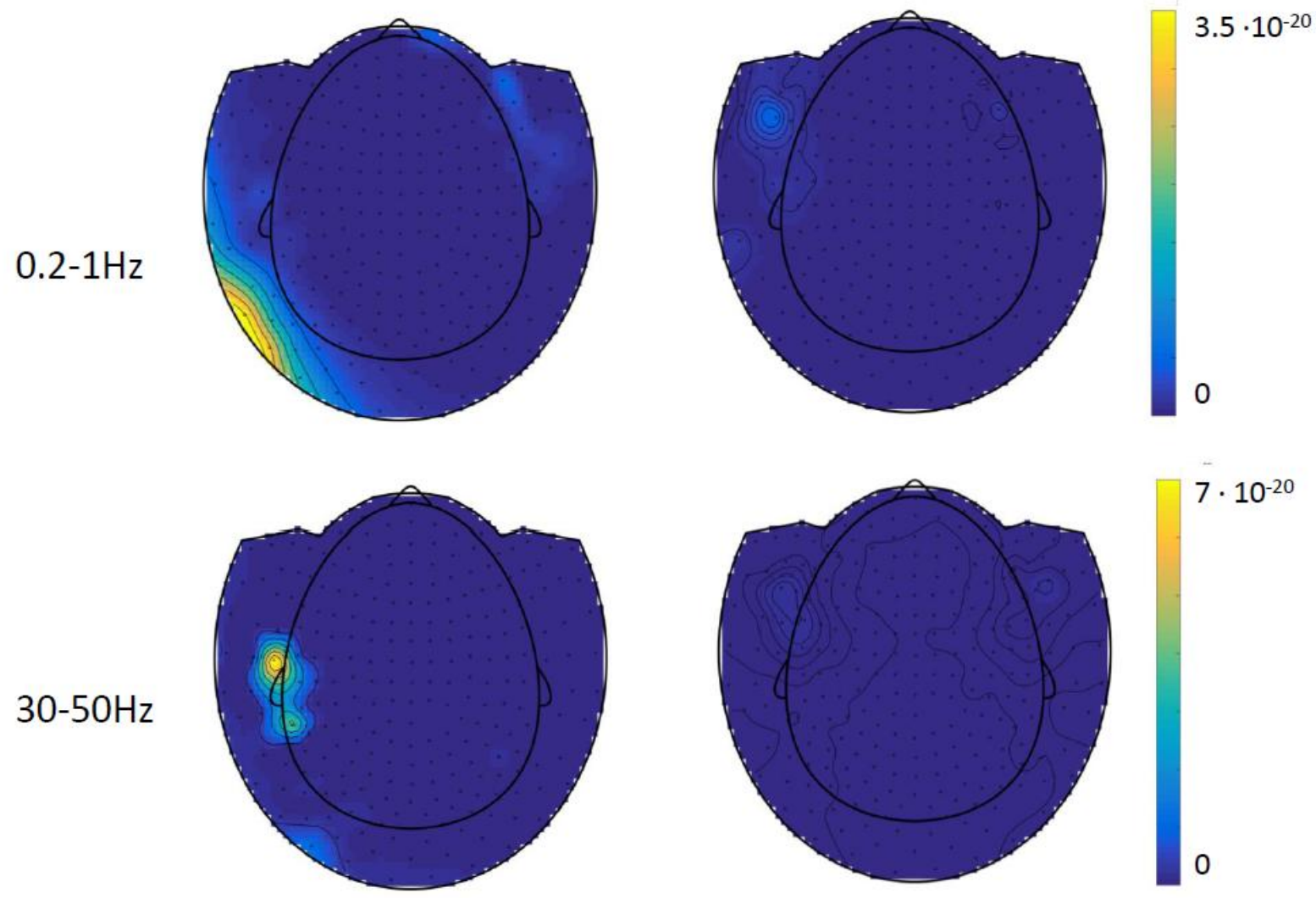

Patient 1

Patient 2

Figure 4: Topographies of the power spectral density frequency maps for two ranges of frequencies: $0.2-1 \mathrm{~Hz}$ for the first line and $30-50 \mathrm{~Hz}$ for the second line. Data from patient 1 are on the left column and data from patient 2 are on the right column. The scales are identical for the two patients. 

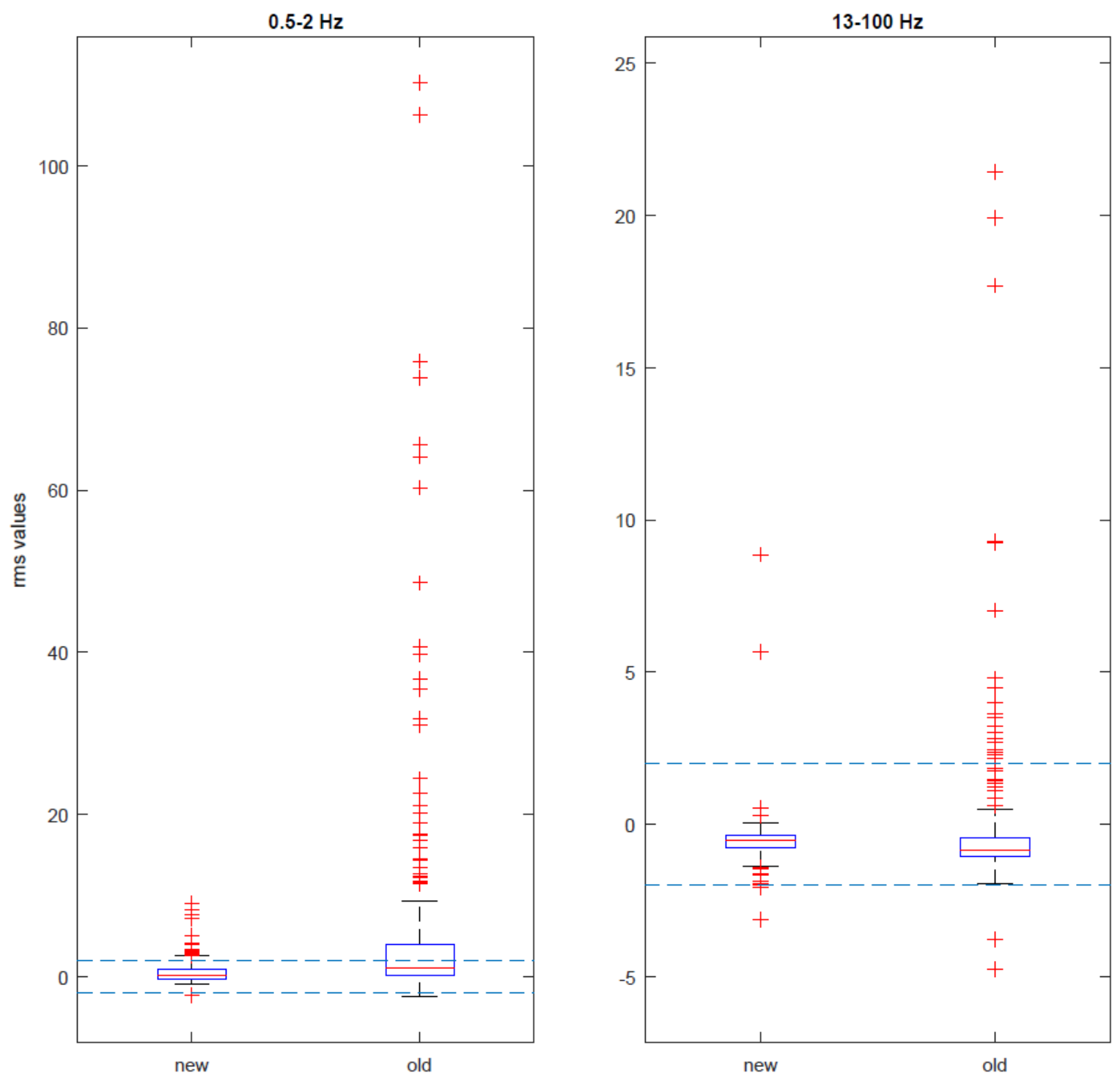

Figure 5: Box-plots of z-scores on signal RMS values across channels for two datasets, on corresponding to the current setup ("new") and the other to the data presented in (Dubarry et al., 2014)) ("old"). The root mean square (RMS) value of each channel filtered in a given frequency band was z-scored with respect to a series of 15 reference datasets coming from patients recorded during presurgical evaluation of epilepsy. The dotted lines correspond to a value of $z= \pm 2$. Red crosses indicate outlier values. 


\section{Acknowledgments}

Part of this work was funded by a joint Agence Nationale de la Recherche (ANR) and Direction Génerale de l'Offre de Santé (DGOS) under grant 'VIBRATIONS' ANR 13 PRTS 0011 01. This work has been carried out within the FHU EPINEXT with the support of the A*MIDEX project (ANR-11IDEX-0001-02) funded by the "Investissements d'Avenir" French Government program managed by the French National Research Agency (ANR), on a platform member of France Life Imaging network (grant ANR-11-INBS-0006).

\section{References}

Baillet S, Mosher J C and Leahy R M 2001 Electromagnetic brain mapping IEEE Signal Processing Magazine 18 14-30

Dalal S S, Baillet S, Adam C, Ducorps A, Schwartz D, Jerbi K, Bertrand O, Garnero L, Martinerie J and Lachaux J P 2009 Simultaneous MEG and intracranial EEG recordings during attentive reading Neuroimage 45 1289-304

Dubarry A S, Badier J M, Trebuchon-Da Fonseca A, Gavaret M, Carron R, Bartolomei F, LiegeoisChauvel C, Regis J, Chauvel P, Alario F X and Benar C G 2014 Simultaneous recording of MEG, EEG and intracerebral EEG during visual stimulation: from feasibility to single-trial analysis Neuroimage 99 548-58

Gavaret M, Dubarry A S, Carron R, Bartolomei F, CG B and Trébuchon A 2016 Simultaneous SEEGMEG-EEG recordings overcome the SEEG limited spatial sampling Epilepsy Res 128 67:72

Kakisaka Y, Kubota Y, Wang Z I, Piao Z, Mosher J C, Gonzalez-Martinez J, Jin K, Alexopoulos A V and Burgess R C 2012 Use of simultaneous depth and MEG recording may provide complementary information regarding the epileptogenic region Epileptic Disord 14 298-303

Koessler L, Cecchin T, Colnat-Coulbois S, Vignal J P, Jonas J, Vespignani H, Ramantani G and Maillard L G 2015 Catching the invisible: mesial temporal source contribution to simultaneous EEG and SEEG recordings Brain Topogr 28 5-20

Lopes da Silva F and Van Rotterdam A 2005 Electroencephalography: basic principles, clinical applications, and related fields, ed Baltimore: Lippincott Williams \& Wilkins)

Malinowska U, Badier J M, Gavaret M, Bartolomei F, Chauvel P and Benar C G 2014 Interictal networks in magnetoencephalography Hum Brain Mapp 35 2789-805

Mikuni N, Nagamine T, Ikeda A, Terada K, Taki W, Kimura J, Kikuchi H and Shibasaki H 1997 Simultaneous recording of epileptiform discharges by MEG and subdural electrodes in temporal lobe epilepsy Neurolmage 5 298-306

Rampp S, Kaltenhauser M, Weigel D, Buchfelder M, Ingmar Blumcke I, Dorfler A and Stefan H 2010 MEG correlates of epileptic high gamma oscillations in invasive EEG Epilepsia $\mathbf{5 1} 1638-42$

Santiuste M, Nowak R, Russi A, Tarancon T, Oliver B, Ayats E, Scheler G and Graetz G 2008 Simultaneous magnetoencephalography and intracranial EEG registration: technical and clinical aspects J Clin Neurophysio/ 25 331-9

Stefan H, Rampp S and Knowlton R C 2011 Magnetoencephalography adds to the surgical evaluation process Epilepsy \& behavior $20172-7$

Sutherling W W, Akhtari M, Mamelak A N, Mosher J, Arthur D, Sands S, Weiss P, Lopez N, DiMauro M, Flynn E and Leah R 2001 Dipole localization of human induced focal afterdischarge seizure in simultaneous magnetoencephalography and electrocorticography Brain Topogr 14 101-16

Urrestarazu E, Chander R, Dubeau F and Gotman J 2007 Interictal high-frequency oscillations (100$500 \mathrm{~Hz}$ ) in the intracerebral EEG of epileptic patients Brain 130 2354-66

Wennberg R, Valiante T and Cheyne D 2011 EEG and MEG in mesial temporal lobe epilepsy: where do the spikes really come from? Clin Neurophysiol 122 1295-313 